\title{
Total angular momentum representation for state-to-state quantum scattering of cold molecules in a magnetic field
}

\author{
Suyesh Koyu, ${ }^{1}$ Rebekah Hermsmeier, ${ }^{1}$ and Timur V. Tscherbul ${ }^{1}$ \\ ${ }^{1}$ Department of Physics, University of Nevada, Reno, NV, 89557, USA
}

(Dated: October 13, 2021)

\begin{abstract}
We show that the integral cross sections for state-to-state quantum scattering of cold molecules in a magnetic field can be efficiently computed using the total angular momentum representation despite the presence of unphysical Zeeman states in the eigenspectrum of the asymptotic Hamiltonian. We demonstrate that the unphysical states arise due to the incompleteness of the space-fixed total angular momentum basis caused by using a fixed cutoff value $J_{\max }$ for the total angular momentum of the collision complex $J$. As a result, certain orbital angular momentum $(l)$ basis states lack the full range of $J$ values required by the angular momentum addition rules, resulting in the appearance of unphysical states. We find that by augmenting the basis with a full range of $J$-states for every $l$, it is possible to completely eliminate the unphysical states from quantum scattering calculations on molecular collisions in external fields. To illustrate the procedure, we use the augmented basis sets to calculate the state-to-state cross sections for rotational and spin relaxation in cold collisions of ${ }^{40} \mathrm{CaH}\left(X^{2} \Sigma^{+}, v=0, N=1, M_{N}=1, M_{S}=1 / 2\right)$ molecules with ${ }^{4} \mathrm{He}$ atoms in a magnetic field. We find excellent agreement with benchmark calculations, validating our proposed procedure. We find that $N$-conserving spin relaxation to the lowest-energy Zeeman state of the $N=1$ manifold, $\left|11 \frac{1}{2}\right\rangle \rightarrow\left|1-1-\frac{1}{2}\right\rangle$ is nearly completely suppressed due to the lack of spin-rotation coupling between the fully spin-stretched Zeeman states. Our results demonstrate the possibility of rigorous, computationally efficient, and unphysical state-free quantum scattering calculations on cold molecular collisions in an external magnetic field.
\end{abstract}




\section{INTRODUCTION}

Ultracold molecular collisions offer a rich playground for exploring and controlling fundamental quantum phenomena in ultracold dilute molecular gases [1]. These fascinating phenomena range from resonant tunneling [2], external field-induced reactive scattering res-

onances $[3,4]$ and long-lived reaction complex formation $[5,6]$ to quantum coherent control [7], many-body entanglement [8], and quantum chaos [9]. In addition, the strong anisotropic dipole-dipole interactions of cold polar molecules are highly tunable by external electric and microwave fields, enabling applications in quantum information processing [10-12], quantum metrology [8] and quantum simulation [13, 14].

First-principles quantum dynamics calculations based on ab initio potential energy surfaces (PESs) [15-30] provide the ultimate theoretical understanding of cold and ultracold molecular collisions allowed by the laws of quantum mechanics. By providing the full stateto-state information about collision observables in the presence of external fields, such calculations can be used to explain experimentally observed phenomena in ultracold molecular gases [22-28] and to predict novel effects in molecular systems not yet realized experimentally [17, 18, 20, 29-36]. Quantum dynamics calculations could be based on either timeindependent or time-dependent scattering formalisms. In the time-dependent approach that has been most widely adopted thus far [15-30] the wavefunction of the collision complex is expanded in a channel basis set [37], and the resulting coupled-channel (CC) equations are solved numerically to generate the full state-to-state scattering $S$-matrix, which provides comprehensive information about the scattering properties (such as the differential/integral cross sections and collision rates) of molecules in different internal states.

The choice of the channel basis is a central aspect of any quantum dynamics calculation, be it time-dependent or time-independent. An efficient basis set results in a compact representation of the scattering wavefunction using the fewest possible basis functions. One such basis set, which proved particularly efficient for molecular collisions in external fields, is based on the total angular momentum representation in either the body-fixed (BF) or space-fixed (SF) coordinate frames [19, 21, 38].

Even though the quantum theory of molecular collisions in external fields was first formulated in the uncoupled SF representation $[15,16]$, it was soon realized that this representation is not computationally efficient for strongly anisotropic atom-molecule and molecule- 
molecule collisions, where the interaction anisotropy couples an exceedingly large number of basis functions, making converged calculations impossible. In contrast, the total angular momentum representation introduced by Arthurs and Dalgarno in 1960 for field-free collisions [39] and by our group in 2010-2012 for collisions in external fields [19, 21, 38], takes into account the approximate conservation of the total angular momentum of the collision complex to drastically reduce the number of basis states required to obtain converged scattering observables. The total angular momentum basis has since been used to obtain converged results for a variety of ultracold atom-molecule [20, 29, 30], molecule-molecule [21], and chemically reactive [3] systems, which had been considered intractable prior to the development of the basis.

A distinctive feature of the total angular momentum basis is the appearance of unphysical Zeeman states of the asymptotic Hamiltonian. As shown in our previous work [19] the unphysical states correspond to the maximum value of the total angular momentum $J_{\max }$ included in the basis set. As a result, transitions involving such states are strongly suppressed at ultralow temperatures, so they can be simply ignored in calculating ultracold collision observables, as was done in Refs. [29, 30, 36]. Nevertheless, the unphysical states tend to congest the spectrum of molecular threshold energies, particularly in the vicinity of nearly degenerate manifolds of excited rotational and/or hyperfine states. This congestion complicates the identification of molecular thresholds and makes it challenging to calculate state-to-state scattering observables in the presence of external fields. It could also cause problems in calculating the near-threshold bound states of collision complexes in the presence of external fields, an essential step in interpreting Feshbach resonances in ultracold atom-molecule $[23,40,41]$ and molecule-molecule $[18,42]$ collisions. Indeed, it is impossible to distinguish between the unphysical and physical states without knowing the exact energies of the latter (used as a benchmark). Thus, while identifying unphysical states in diatomic molecules is a relatively straightforward task, it is far from trivial for the near-threshold bound states of atom-molecule and molecule-molecule complexes, motivating an in-depth study into the origin of unphysical states, and the development of efficient strategies for their elimination.

Here, we present such a study using a space-fixed (SF) total angular momentum representation of the scattering Hamiltonian in the presence of an external magnetic field. This representation clarifies the symmetry of the asymptotic Hamiltonian with respect to the or- 
bital motion of the atom around the diatomic molecule, as a result of which the Hamiltonian commutes with $\hat{L}^{2}$, the squared orbital angular momentum operator of the collision complex. This results in a representation, which block-diagonalizes the matrix of the asymptotic Hamiltonian, and clarifies the origin of the unphysical states as due to the incompleteness of the SF total angular momentum basis. This allows us to pinpoint the basis states responsible for the formation of unphysical states, and to suggest a procedure for completing the SF basis, leading to a general strategy for eliminating the unphysical states from quantum scattering calculations on molecular collisions in magnetic fields.

To illustrate the basis set completion procedure, we calculate the collision energy dependence of state-to-state scattering cross sections for cold collisions of rotationally excited $\mathrm{CaH}$ molecules with ${ }^{4} \mathrm{He}$ atoms in a magnetic field using the augmented SF basis. Our results are in excellent agreement with benchmark calculations based on the fully uncoupled SF representation $[15,16]$ for all final states over a wide region of collision energies, demonstrating the feasibility of unphysical state-free quantum scattering calculations with a negligible computational overhead for low-lying rotational states.

This article is organized as follows. Section IIA presents the necessary background information on the total angular momentum representation for molecular collisions in a magnetic field. In Sec. IIB we consider the origin of unphysical states in the SF total angular momentum representation, and describe a basis set completion procedure to arrive at an unphysical-state-free, augmented SF representation of the asymptotic Hamiltonian. Section IIIA illustrates the procedure by constructing the augmented SF basis for a ${ }^{2} \Sigma$ molecule in a magnetic field. Quantum scattering calculations of state-to-state cross sections for cold

${ }^{4} \mathrm{He}+{ }^{40} \mathrm{CaH}$ collisions in the augmented SF basis are presented and compared with benchmark results in Sec. IIIB. Finally, Sec. IV summarizes our main findings and outlines a few promising directions of future research.

\section{THEORY}

\section{A. Total angular momentum basis for collisions in an external magnetic field}

Here, we will only give the essential details of the total angular momentum representation for molecular collisions in a magnetic field, which are required to introduce the concept of 
unphysical states. The reader is referred to Refs. [19, 21] for a detailed description of the theory and computational methodology.

In the remainder of this paper, we will focus on the case of cold collisions of ${ }^{2} \Sigma$ molecules with spherically symmetric atoms in the ${ }^{1} \mathrm{~S}$ electronic state. Owing to its simplicity, this case is ideally suited to illustrate the main idea of our approach, which can be extended to molecule-molecule collisions and chemical reactions in a magnetic field following [3, 19, 21].

Our goal is to solve the time-independent Schrödinger equation for the collision complex formed by a structureless atom and a ${ }^{2} \Sigma$ molecule and described by the Hamiltonian

$$
\hat{H}=-\frac{1}{2 \mu R} \frac{d^{2}}{d R^{2}} R+\frac{\hat{L}^{2}}{2 \mu R^{2}}+\hat{H}_{\mathrm{mol}}+\hat{V}(R, \theta),
$$

subject to the scattering boundary conditions. Here, $R$ is the magnitude of the Jacobi vector $\mathbf{R}$ from the center of mass of the diatomic molecule to the atom, $\theta$ is the Jacobi angle between $\mathbf{R}$ and $\mathbf{r}$, the internuclear separation vector of the molecule, $\mu$ is the reduced mass and $\hat{L}$ is the orbital angular momentum of the atom-molecule system. Furthermore, $\hat{V}$ is the atom-molecule interaction potential, and $\hat{H}_{\text {mol }}$ is the Hamiltonian that describes the ${ }^{2} \Sigma$ molecule's internal structure, which includes the rotational structure, the spin-rotation interaction, and the Zeeman effect

$$
\hat{H}_{\mathrm{mol}}=B_{e} \hat{N}^{2}+\gamma_{\mathrm{sr}} \hat{\mathbf{N}} \cdot \hat{\mathbf{S}}+2 \mu_{0} B \hat{S}_{Z}
$$

where $\hat{\mathbf{N}}$ is the rotational angular momentum of the diatomic molecule, $\hat{\mathbf{S}}$ is the electronic spin, $B_{e}$ is the rotational constant, $\gamma_{\mathrm{sr}}$ is the spin-rotation constant, $\mu_{0}$ is the Bohr magneton, and $B$ is the magnitude of the external magnetic field, which defines the SF quantization axis. We neglect the hyperfine structure, which is a good approximation in the large-field limit [29], and assume that the internuclear distance of the diatomic molecule is fixed at its equilibrium value (the rigid rotor approximation).

To solve the time-independent Schrödinger equation with the Hamiltonian (1) we expand the wavefunction in the basis set [19]

$$
|\Psi\rangle=\frac{1}{R} \sum_{n} F_{n}(R)\left|\Phi_{n}\right\rangle,
$$

where $\left|\Phi_{n}\right\rangle$ are the angular basis functions and $F_{n}^{M}(R)$ are the radial expansion coefficients.

We choose the angular basis functions to be the eigenstates of $|\hat{\mathbf{J}}|^{2}$ and $\hat{J}_{Z}$, where $\hat{\mathbf{J}}$ is the total angular momentum of the collision complex, and $\hat{J}_{Z}$ is its projection on the SF 
quantization axis defined by the external (magnetic) field. As shown in our previous work, the SF total angular momentum basis has excellent convergence properties, allowing rigorous numerical treatment of strongly anisotropic molecular collisions [19-21] and chemical reactions [3] in the presence of external dc electric and/or magnetic fields.

To construct the SF total angular momentum basis functions, we first couple $\mathbf{N}$ and $\mathbf{S}$ form the total angular momentum of the isolated ${ }^{2} \Sigma$ molecule $\hat{\mathbf{j}}$, and then couple $\hat{\mathbf{j}}$ with $\hat{\mathbf{L}}$ to form $\hat{\mathbf{J}}$

$$
\left|\Phi_{n}\right\rangle=|J M l j(N S)\rangle=\sum_{m_{j}, m_{l}}\left\langle j m_{j}, l m_{l} \mid J M\right\rangle\left|j m_{j}(N S)\right\rangle\left|l m_{l}\right\rangle
$$

where where $\left|j m_{j}(N S)\right\rangle=\sum_{N, M_{N}}\left\langle N M_{N} S M_{S} \mid j m_{j}\right\rangle\left|N M_{N}\right\rangle\left|S M_{S}\right\rangle$ are the eigenstates of $|\hat{\mathbf{j}}|^{2}$ and $\hat{j}_{Z},\left|N M_{N}\right\rangle$ are those of $|\hat{\mathbf{N}}|^{2}$ and $\hat{N}_{Z}$, and $\left|S M_{S}\right\rangle$ are those of $|\hat{\mathbf{S}}|^{2}$ and $\hat{S}_{Z}$ (for ${ }^{2} \Sigma$ molecules considered here, $S=1 / 2)$, and $\left\langle j_{1} m_{1} j_{2} m_{2} \mid j m\right\rangle$ are the Clebsch-Gordan coefficients.

The radial expansion coefficients $F_{n}(R)$ in Eq. (3) can be obtained by solving the CC equations

$$
\left[\frac{d^{2}}{d R^{2}}+2 \mu E\right] F_{n}(R)=2 \mu \sum_{n^{\prime}}\left\langle n\left|\hat{V}(R, \theta)+\frac{\hat{L}^{2}}{2 \mu R^{2}}+\hat{H}_{\mathrm{mol}}\right| n^{\prime}\right\rangle F_{n^{\prime}}(R)
$$

where $|n\rangle$ denote the coupled basis states $|J M l j(N S)\rangle$. The asymptotic behavior of the solutions in the limit $R \rightarrow \infty$ defines the scattering $S$-matrix [19] for a given value of the total angular momentum projection onto the field axis $M$, from which the integral cross sections are evaluated as

$$
\sigma_{\gamma \rightarrow \gamma^{\prime}}=\frac{2 \pi}{k_{\gamma}^{2}} \sum_{M} \sum_{l, l^{\prime}}\left|\delta_{\gamma \gamma^{\prime}} \delta_{l l^{\prime}}-S_{\gamma l, \gamma^{\prime} l^{\prime}}^{M}\right|^{2}
$$

where $\gamma$ and $\gamma^{\prime}$ are the internal states of the colliding molecule (or eigenstates of $\hat{H}_{\text {mol }}$ ), among which collision-induced transitions occur.

The CC equations are parametrized by the matrix elements of the molecular Hamiltonian $\hat{H}_{\text {mol }}$, the interaction potential $\hat{V}(R, \theta)$, and the angular kinetic energy $\hat{L}^{2} /\left(2 \mu R^{2}\right)$. Below we present the expressions for these matrix elements in the SF total angular momentum basis, derived using standard techniques of angular momentum theory [43] 
We start from the Zeeman matrix elements

$$
\begin{aligned}
& \left\langle J M l j(N S)\left|2 \mu_{0} B \hat{S}_{Z}\right| J^{\prime} M^{\prime} l^{\prime} j^{\prime}\left(N^{\prime} S^{\prime}\right)\right\rangle=\delta_{l l^{\prime}} \delta_{N N^{\prime}}(-1)^{J-M+J^{\prime}+l+2 j+N+S+2}\left[(2 J+1)\left(2 J^{\prime}+1\right)\right. \\
& \left.\times\left(2 j^{\prime}+1\right)(2 j+1)(2 S+1) S(S+1)\right]^{1 / 2} 2 \mu_{0} B\left(\begin{array}{ccc}
J & 1 & J^{\prime} \\
-M & 0 & M
\end{array}\right)\left\{\begin{array}{lll}
j & j^{\prime} & 1 \\
J^{\prime} & J & l
\end{array}\right\}\left\{\begin{array}{lll}
S & S & 1 \\
j^{\prime} & j & N
\end{array}\right\}, \quad(7)
\end{aligned}
$$

where the symbols in parentheses and figure brackets are the $3-j$ and $6-j$ symbols. Note that the interaction with external magnetic fields couples the states with different total angular momenta $J$, a consequence of the breaking of rotational invariance in the presence of external fields. The matrix elements of the rotational kinetic energy of the diatomic molecule are diagonal in the SF total angular momentum coupled basis

$$
\left\langle J M l j(N S)\left|B_{e} \hat{N}^{2}\right| J^{\prime} M^{\prime} l^{\prime} j^{\prime}\left(N^{\prime} S^{\prime}\right)\right\rangle=B_{e} N(N+1) \delta_{J J^{\prime}} \delta_{M M^{\prime}} \delta_{l l^{\prime}} \delta_{j j^{\prime}} \delta_{N N^{\prime}} \delta_{S S^{\prime}}
$$

as are the matrix elements of the spin-rotation matrix interaction (since our angular basis functions are the eigenstates of $\hat{j}^{2}$

$$
\begin{aligned}
\left\langle J M l j(N S)\left|\gamma_{\mathrm{sr}} \hat{\mathbf{N}} \cdot \hat{\mathbf{S}}\right| J^{\prime} M^{\prime} l^{\prime} j^{\prime}\left(N^{\prime} S^{\prime}\right)\right\rangle= & \delta_{J J^{\prime}} \\
& \delta_{M M^{\prime}} \delta_{l l^{\prime}} \delta_{j j^{\prime}} \delta_{N N^{\prime}} \delta_{S S^{\prime}} \\
& \times \frac{1}{2} \gamma_{\mathrm{sr}}[j(j+1)-N(N+1)-S(S+1)]
\end{aligned}
$$

Finally, the matrix elements of the atom-molecule interaction potential are diagonal in $J$ as a consequence of rotational invariance of intermolecular interactions

$$
\begin{aligned}
& \left\langle J M l j(N S)|V(R, \theta)| J^{\prime} M^{\prime} l^{\prime} j^{\prime}\left(N^{\prime} S^{\prime}\right)\right\rangle=\delta_{J J^{\prime}} \delta_{M M^{\prime}} \delta_{S S^{\prime}}(-1)^{J+S+2 j^{\prime}+\lambda}\left[\left(2 j^{\prime}+1\right)(2 j+1)\right. \\
& \left.\times(2 N+1)\left(2 N^{\prime}+1\right)(2 l+1)\left(2 l^{\prime}+1\right)\right]^{1 / 2} \sum_{\lambda} V_{\lambda}(R)\left\{\begin{array}{ccc}
N & j & S \\
j^{\prime} & N^{\prime} & \lambda
\end{array}\right\}\left\{\begin{array}{lll}
j & l & J \\
l^{\prime} & j^{\prime} & \lambda
\end{array}\right\}\left(\begin{array}{ccc}
N & \lambda & N^{\prime} \\
0 & 0 & 0
\end{array}\right)\left(\begin{array}{lll}
l & \lambda & l^{\prime} \\
0 & 0 & 0
\end{array}\right),
\end{aligned}
$$

where $V_{\lambda}(R)$ are the radial expansion coefficients of the atom-molecule interaction PES in Legendre polynomials, $V(R, \theta)=\sum_{\lambda} V_{\lambda}(R) P_{\lambda}(\cos \theta)$.

\section{B. Unphysical states in the SF representation and their elimination}

Previous theoretical work has focused on the unphysical states arising in the BF formulation of the collision problem [19]. To gain additional insight into the origin of unphysical 
states, here we reformulate the problem in the SF total angular momentum representation. As shown below, this reformulation makes explicit the symmetry of the asymptotic Hamiltonian related to to the conservation of the squared orbital angular momentum of the collision complex $\hat{L}^{2}$. This allows us to identify the basis states responsible for the formation of unphysical states and to suggest a general strategy for eliminating these states from quantum scattering calculations on molecular collisions in external fields.

Consider the matrix of the asymptotic Hamiltonian (7) in the SF total angular momentum representation. Restricting our attention for the moment to the ground rotational state $(N=0, j=1 / 2)$, we choose a minimal basis consisting of two total angular momentum states, $J=1 / 2$ and $J=3 / 2$. The corresponding values of $l$ range from $|j-J|$ to $|j+J|$. Using the shorthand notation $|J l j\rangle$ for the basis states $|J l j(N S)\rangle$ within the $N=0$ and $S=1 / 2$ subspace of interest, there are two basis states, $\left|\frac{1}{2} 0 \frac{1}{2}\right\rangle$ and $\left|\frac{1}{2} 1 \frac{1}{2}\right\rangle$ for $J=1 / 2$ and two basis states, $\left|\frac{3}{2} 1 \frac{1}{2}\right\rangle$ and $\left|\frac{3}{2} 2 \frac{1}{2}\right\rangle$ for $J=3 / 2$. The asymptotic Hamiltonian is represented in this basis by a $4 \times 4$ matrix (with the basis functions arranged in the sequence $\left|\frac{1}{2} 0 \frac{1}{2}\right\rangle$, $\left.\left|\frac{1}{2} 1 \frac{1}{2}\right\rangle,\left|\frac{3}{2} 1 \frac{1}{2}\right\rangle,\left|\frac{3}{2} 2 \frac{1}{2}\right\rangle\right)$

$$
\hat{H}_{\text {mol }}=\left[\begin{array}{cccc}
\mu_{0} B & 0 & 0 & 0 \\
0 & -\frac{1}{3} \mu_{0} B & \frac{2 \sqrt{2}}{3} \mu_{0} B & 0 \\
0 & \frac{2 \sqrt{2}}{3} \mu_{0} B & \frac{1}{3} \mu_{0} B & 0 \\
0 & 0 & 0 & -\frac{1}{5} \mu_{0} B
\end{array}\right]
$$

This matrix is diagonal in $l$ as a consequence of $\hat{L}^{2}$ commuting with $\hat{H}_{\text {mol }}$, and thus the matrix representation (11) is notably simpler than that of $\hat{H}_{\mathrm{mol}}$ in the BF total angular momentum basis [19] (where it is given by a full matrix). The block-diagonal structure of the matrix permits analytical diagonalization to yield the eigenvalues

$$
\lambda_{1}=\lambda_{2}=\mu_{0} B, \quad \lambda_{3}=-\mu_{0} B, \quad \lambda_{4}=-\frac{1}{5} \mu_{0} B
$$

and the corresponding eigenvectors

$$
\begin{aligned}
& |1\rangle=|J=1 / 2, l=0, j=1 / 2\rangle \\
& |2\rangle=\frac{1}{\sqrt{3}}|J=1 / 2, l=1, j=1 / 2\rangle+\sqrt{\frac{2}{3}}|J=3 / 2, l=1, j=1 / 2\rangle \\
& |3\rangle=-\sqrt{\frac{2}{3}}|J=1 / 2, l=1, j=1 / 2\rangle+\frac{1}{\sqrt{3}}|J=3 / 2, l=1, j=1 / 2\rangle \\
& |4\rangle=|J=3 / 2, l=2, j=1 / 2\rangle
\end{aligned}
$$


We observe that three of the eigenvalues correspond to the physical Zeeman states of a rotationless ${ }^{2} \Sigma$ molecule in a magnetic field $\left( \pm \mu_{0} B\right)$, whereas the eigenvalue $\lambda_{4}$ is unphysical. By examining the $1 \times 1$ subblock of the asymptotic Hamiltonian matrix in Eq. (11) that corresponds to $l=2$, we notice that it is spanned by a single basis state $\left|\frac{3}{2} 2 \frac{1}{2}\right\rangle$ with $\lambda_{4}=$ $\left\langle\frac{3}{2} 2 \frac{1}{2}\left|\hat{H}_{\text {mol }}\right| \frac{3}{2} 2 \frac{1}{2}\right\rangle$. Significantly, this is not a complete basis for $l=2$ : the state $\left|\frac{5}{2} 2 \frac{1}{2}\right\rangle$ is missing because the SF basis set $|J l j(N S)\rangle$ defined above only includes (by construction) the two lowest values of $J=1 / 2$ and $3 / 2$. This is reflected in the absence of the contribution from basis state $\left|\frac{5}{2} 2 \frac{1}{2}\right\rangle$ in the eigenvector $|4\rangle[\mathrm{Eq}$. (13)]. It is now clear that the unphysical state $|4\rangle$ arises from the incompleteness of the $l=2$ basis set caused by the truncation procedure, which limits the range of available $J$ states to $1 / 2$ and $3 / 2$.

The above arguments suggest that, in order to eliminate unphysical states from the spectrum of the asymptotic Hamiltonian, we can simply remove the unphysical $1 \times 1$ subblock corresponding to $l=2$ in Eq. (11) by excluding the state $\left|\frac{3}{2} 2 \frac{1}{2}\right\rangle$ from the basis set. Alternatively, we could complete the $l=2$ subblock by including the basis state $\left|\frac{5}{2} 2 \frac{1}{2}\right\rangle$. The former procedure simply eliminates the unphysical eigenvalue $\lambda_{4}$, whereas the latter results in the following augmented matrix of the asymptotic Hamiltonian (with the basis functions arranged as $\left.\left|\frac{1}{2} 0 \frac{1}{2}\right\rangle,\left|\frac{1}{2} 1 \frac{1}{2}\right\rangle,\left|\frac{3}{2} 1 \frac{1}{2}\right\rangle,\left|\frac{3}{2} 2 \frac{1}{2}\right\rangle,\left|\frac{5}{2} 2 \frac{1}{2}\right\rangle\right)$

$$
\hat{H}_{m o l}=\left[\begin{array}{ccccc}
\mu_{0} B & 0 & 0 & 0 & 0 \\
0 & -\frac{1}{3} \mu_{0} B & \frac{2 \sqrt{2}}{3} \mu_{0} B & 0 & 0 \\
0 & \frac{2 \sqrt{2}}{3} \mu_{0} B & \frac{1}{3} \mu_{0} B & 0 & 0 \\
0 & 0 & 0 & -\frac{1}{5} \mu_{0} B & \frac{2 \sqrt{6}}{5} \mu_{0} B \\
0 & 0 & 0 & \frac{2 \sqrt{6}}{5} \mu_{0} B & \frac{1}{5} \mu_{0} B
\end{array}\right]
$$

The $2 \times 2$ block corresponding to $l=2$ is now complete, and its eigenvalues are

$$
\lambda_{4}=\mu_{0} B, \quad \lambda_{5}=-\mu_{0} B
$$

with the corresponding eigenvectors

$$
\begin{aligned}
& |4\rangle=\sqrt{\frac{2}{5}}|J=3 / 2, l=2, j=1 / 2\rangle+\sqrt{\frac{3}{5}}|J=5 / 2, l=2, j=1 / 2\rangle \\
& |5\rangle=-\sqrt{\frac{3}{5}}|J=3 / 2, l=2, j=1 / 2\rangle+\sqrt{\frac{2}{5}}|J=5 / 2, l=2, j=1 / 2\rangle
\end{aligned}
$$

We observe that the unphysical states have disappeared as expected, since the matrix representation of the asymptotic Hamiltonian in the $l=2$ sector is now complete. 

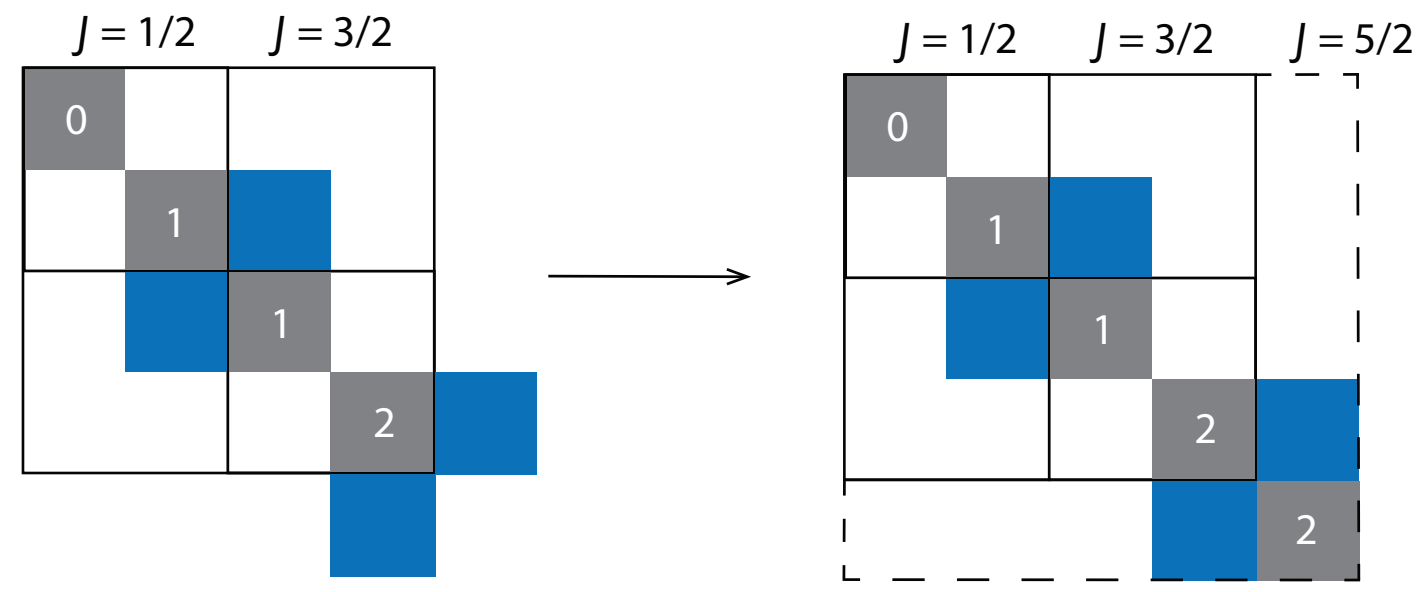

FIG. 1. Schematic illustration of the basis set completion procedure used to construct the augmented SF basis. (Left): Matrix representation of the asymptotic Hamiltonian in the SF total angular momentum basis truncated using the standard procedure. Only the $N=0(j=1 / 2)$ basis states are included, giving rise to two $l$ basis states for each $J$ with $l=J \pm 1 / 2$. The Zeeman Hamiltonian is diagonal in $l$ but off-diagonal in $J$ (see text). The values of $J$ corresponding to each basis function are indicated at the top of each block; the diagonal blocks are labeled by the orbital angular momentum quantum number $l$. The diagonal (off-diagonal) magnetic field couplings are represented by grey (blue) squares. (Right): Matrix representation of the asymptotic Hamiltonian in the augmented $\mathrm{SF}$ basis including the $l=2, J=5 / 2$ state required to obtain the complete angular momentum basis set for $l=2$. No unphysical states occur in the augmented SF representation.

We now generalize the above discussion to the case of arbitrary SF basis sets $|J M l j(N S)\rangle$ with the goal of devising a general procedure for eliminating unphysical Zeeman states. As we have just seen, the origin of these states can be attributed to the incompleteness of the $|J M l j(N S)\rangle$ basis truncated at a finite $J$ that is below the maximum value allowed by the angular momentum addition rules. As shown in Fig. 1, conventional truncation of the basis set at a predetermined cutoff value of $J=J_{\max }$ leaves out the important fieldinduced couplings between the states of different $J$ but same $l$, thereby producing unphysical Zeeman states. Therefore, in order to eliminate the unphysical states, we need to remove the "dangling" field couplings.

Two general strategies for achieving this are immediately apparent. First, as observed 
above, one can exclude the $|J M l j(N S)\rangle$ basis states with the $l$ values, for which $j+l \geq J_{\max }$. Test calculations show that the exclusion strategy does not produce meaningful results, which suggests that the excluded states play a non-negligible role in collision dynamics. An alternative idea, illustrated in Fig. 1, is to complete the SF basis for each $l$ by including the full range of $J$ values up to $J_{\max }(j, l)=j+l$. We will refer to such a basis as the augmented $S F$ basis. We note that the completion procedure can quickly become computationally expensive because $J_{\max }(j, l)=j+l$ can become very large for high $j$, thereby defeating the purpose of using the total angular momentum representation (which saves computational time by including a limited number of $J$ states with $J<J_{\max }$ ) [19]. As a result, the completion procedure should be limited to low $N$ and $j$ values (i.e., those in the vicinity of the collision threshold) to prevent rapid growth of the basis set.

To implement the basis set completion procedure in practice, we proceed to define and truncate the augmented SF total angular momentum basis using the following algorithm, assuming that the values of molecular spin $\left(S=1 / 2\right.$ for ${ }^{2} \Sigma$ molecules) and the upper bounds $N_{\max }$ and $J_{\max }$ are supplied as input parameters

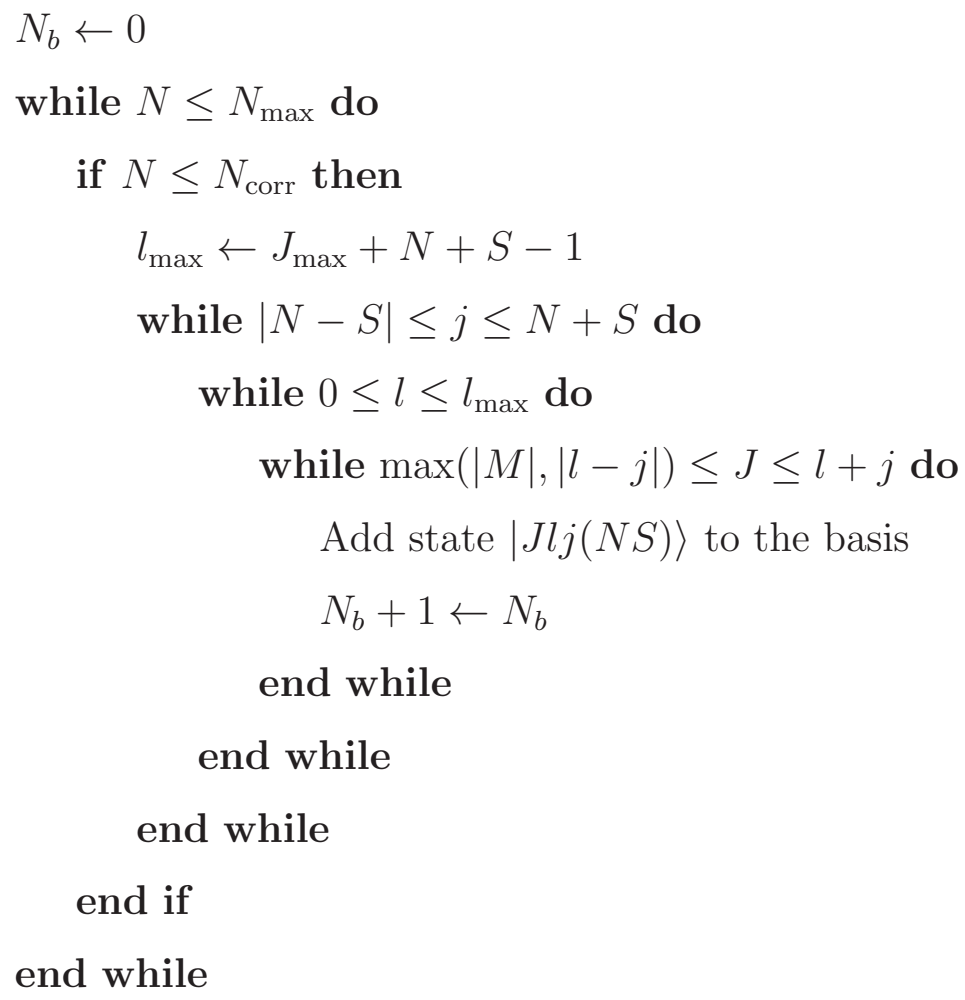

The outer loop runs over the rotational quantum numbers $N$ and the first if statement ensures that the augmented SF basis is constructed only for a subset of $N$ values of interest 
( $N \leq N_{\text {corr }}$, where $N_{\text {corr }}$ is the number of channels, for which unphysical states are to be eliminated, e.g., near-threshold or open channels). In the next step, we define the maximum orbital angular momentum quantum number $l_{\max }$ such that it does not exceed the maximum possible value allowed by angular momentum addition rules $\left(J_{\max }+N+S\right)$. Finally, we construct a loop over the total angular momentum quantum number $J$ in such a way as to ensure basis set completeness for each $l$ as described above.

\section{RESULTS AND DISCUSSION}

In the preceding section, we have described a basis set completion procedure to construct the augmented SF total angular momentum representation, which is free from unphysical states. Here, we illustrate the procedure by calculating the spectrum of the asymptotic Hamiltonian of the $\mathrm{CaH}$ molecule in a magnetic field. We also test the accuracy of the augmented SF basis by carrying out quantum scattering calculations on cold $\mathrm{He}+\mathrm{CaH}$ collisions in a magnetic field.

\section{A. Unphysical state-free representation: Basis set completion procedure and aug-} mented SF total angular momentum representation

Figures 2(a)-(d) show the eigenspectrum of the asymptotic Hamiltonian as a function of external magnetic field calculated for a prototypical ${ }^{2} \Sigma$ molecule $\mathrm{CaH}\left({ }^{2} \Sigma^{+}\right)$using the standard SF basis set $|J M l j(N S)\rangle$ truncated at $J_{\max }=7 / 2$. While there is only a single unphysical Zeeman state in the ground $N=0$ manifold, there are 9 such states in the $N=1$ manifold (shown in Fig. 2(a) by the green lines). The fraction of unphysical states $n_{u} /\left(n_{p}+n_{u}\right)$, where $n_{u}\left(n_{p}\right)$ is the number of unphysical (physical) Zeeman levels, grows rapidly with $N$ as shown in Fig. 2(e). Even though the growth is less steep for higher $J_{\max }$, we observe that most of the high- $N$ eigenstates of $\hat{H}_{\text {mol }}$ are unphysical for $J_{\max } \leq 7.5$.

The physical Zeeman states in the strong field limit are well approximated by the fully uncoupled basis states $\left|N M_{N} M_{S}\right\rangle$ (see Sec. II above), where $N$ is the rotational quantum number, and $M_{N}$ and $M_{S}$ are the projections of $\mathbf{N}$ and $\mathbf{S}$ onto the magnetic field axis.

We note that the Zeeman sublevels $|5\rangle$ and $|8\rangle$ in the $N=1$ manifold are special in that they correspond to the fully spin-stretched states $\left|1-1-\frac{1}{2}\right\rangle$ and $\left|11 \frac{1}{2}\right\rangle$ that are are 

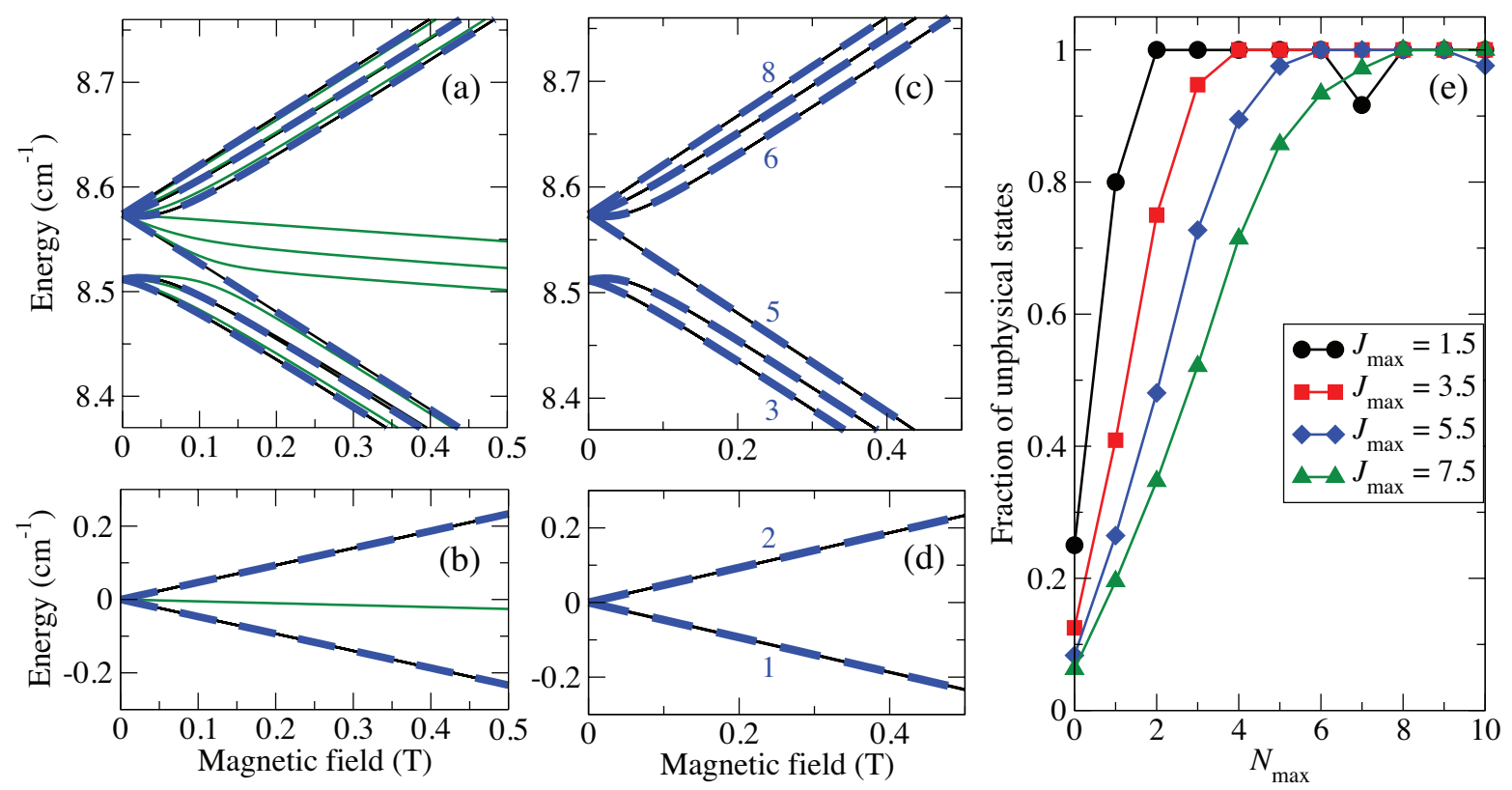

FIG. 2. Eigenvalues of the asymptotic Hamiltonian for $\mathrm{CaH}\left({ }^{2} \Sigma^{+}\right)$in the $N=1$ (a) and $N=0$ (b) rotational states plotted as a function of external magnetic field. The SF basis used to compute the eigenvalues includes all $J$ states with $J \leq J_{\max }=7 / 2$. The physical Zeeman levels of $\mathrm{CaH}\left({ }^{2} \Sigma^{+}\right)$are shown by dashed lines; unphysical Zeeman levels are shown by green lines. (c), (d) Same as panels (a), (b) but computed using the augmented SF basis (see text). Note the absence of unphysical states in the latter basis.

not affected by the spin-rotation interaction. As such, $M_{S}$ and $M_{N}$ remain rigorously good quantum numbers for these states at all magnetic fields. In contrast, the other $N=1$ Zeeman states are coupled by the spin-rotation interaction, resulting in substantial mixing between their $M_{S}$ and $M_{N}$ components. For example, $|6\rangle=0.94\left|1-1 \frac{1}{2}\right\rangle+0.33\left|10-\frac{1}{2}\right\rangle$ at $B=0.1 \mathrm{~T}$. This mixing is large in $\mathrm{CaH}$ due to its large spin-rotation constant, and will generally be much weaker in heavier molecules with smaller spin-rotation constants such as $\mathrm{YbF}[40]$ at the same magnetic field, resulting in purer $\left|N M_{N} M_{S}\right\rangle$ states.

Returning to the discussion of unphysical states, we observe from Fig. 2(a), that some unphysical states can lie very close in energy to the true physical states (such as state |8〉). As stated in the Introduction, this could lead to difficulties in calculating state-tostate scattering cross sections and near-threshold bound states of atom-molecule complexes in external fields, motivating the search for an unphysical state-free representation of the 
asymptotic Hamiltonian.

In Figs. 2(c) and (d) we plot the spectrum of the asymptotic Hamiltonian calculated using the augmented SF total angular momentum basis for $N=0$ and 1 constructed as described in Sec. IIB. We observe that the spectrum is completely free of unphysical states, as expected for a complete angular momentum basis set (see the previous section). This shows that the augmented SF basis provides an appealing, unphysical states-free representation of the asymptotic Hamiltonian.

\section{B. State-to-state $\mathrm{He}-\mathrm{CaH}$ scattering in a magnetic field}

In this section, we will validate the augmented SF total angular momentum basis proposed in Sec. IIB. To this end, we compare state-to-state cross sections for ${ }^{40} \mathrm{He}+{ }^{40} \mathrm{CaH}$ collisions calculated using the new basis with benchmark results obtained using the fully uncoupled SF representation $[15,16]$. To obtain converged solutions of the time-independent Schrödinger equation (1) we propagate the CC equations on the radial grid from $R=2 a_{0}$ to $R=60 a_{0}$ with the grid step of $0.04 a_{0}$, which produces the integral cross sections converged to $<5 \%$. The total angular momentum basis sets included all states with $N \leq 5$ and $J \leq 6.5$. We use a highly accurate $a b$ initio He-CaH PES developed in Ref. [44] and employed in a number of low-temperature scattering calculations [19, 35, 45-48]. The PES is expanded in Legendre polynomials as $V(R, \theta)=\sum_{\lambda=0}^{12} V_{\lambda}(R) P_{\lambda}(\cos \theta)$ and the radial coefficients $V_{\lambda}(R)$ in Eq. (10) are evaluated on a 40-point Gauss-Legendre quadrature. The convergence parameters used in the benchmark calculations are the same as (or more extensive than) those used in our previous work [19].

Figure 3 shows the collision energy dependence of state-to-state cross sections for ${ }^{40} \mathrm{He}+{ }^{40} \mathrm{CaH}$ collisions calculated using the augmented SF total angular momentum basis vs. the benchmark values $[15,16]$. The cross sections calculated using two completely

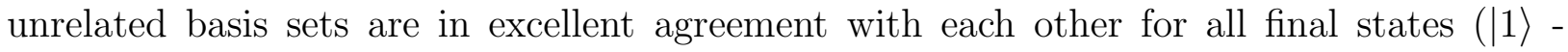
$|8\rangle)$ and collision energies. Significantly, the agreement does not deteriorate near scattering resonances near $0.5 \mathrm{~K}$, which are very sensitive to fine details of collision dynamics. These results strongly suggest that the augmented SF total angular momentum representation provides an accurate description of quantum state-to-state collision dynamics.

As shown previously [35] collisions with cold He atoms result in rapid rotational relaxation 


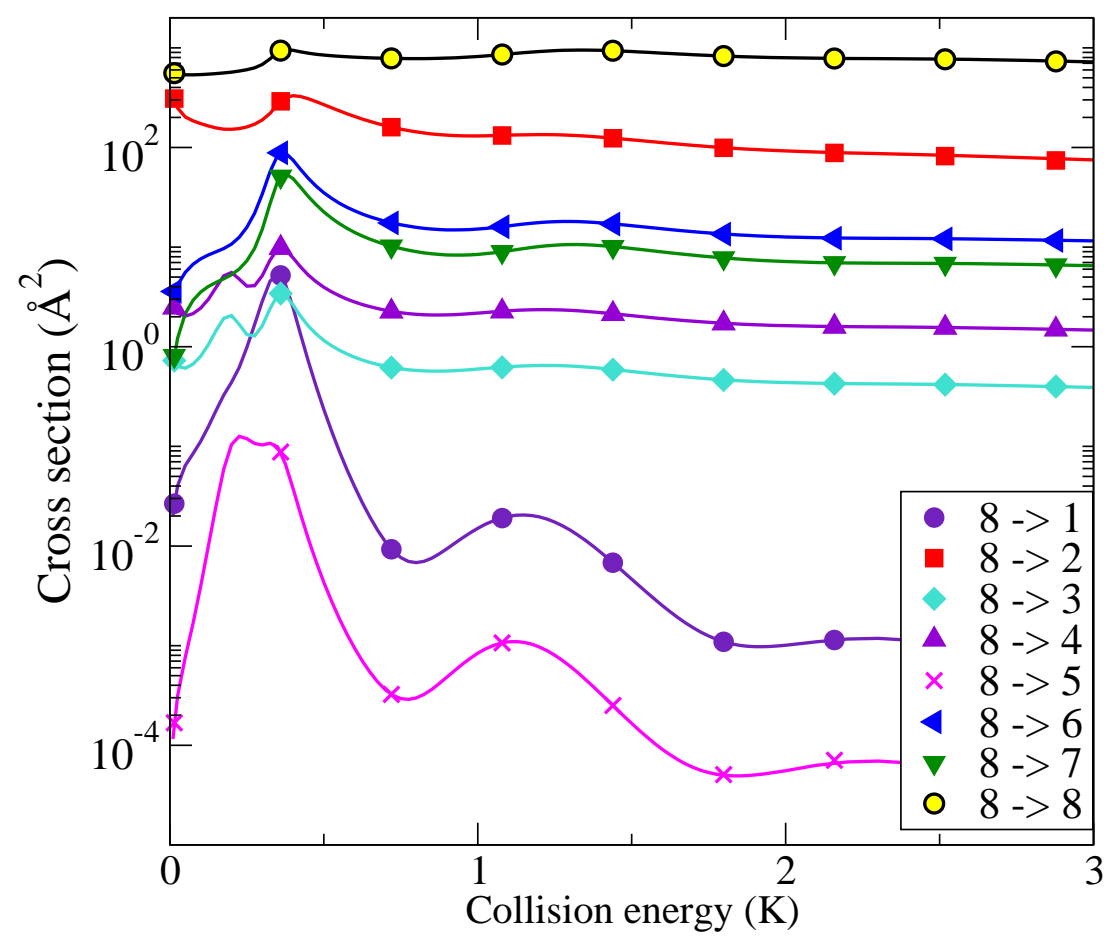

FIG. 3. Collision energy dependence of state-to-state integral cross sections for cold ${ }^{4} \mathrm{He}+{ }^{40} \mathrm{CaH}$ collisions in a magnetic field of $0.1 \mathrm{~T}$ with ${ }^{40} \mathrm{CaH}$ molecules initially in the fully spin-stretched rotationally excited state $|8\rangle=\left|11 \frac{1}{2}\right\rangle$. Symbols - present calculations using the augmented SF total angular momentum basis with $J_{\max }=6.5$. Full lines - benchmark calculations based on the uncoupled space-fixed representation [16, 35]. See Figs. 2(c)-(d) for the Zeeman diagram of CaH showing the final states $|1\rangle-|8\rangle$.

of $\mathrm{CaH}$ molecules, which transfers molecular population from the rotationally excited initial state $|8\rangle$ to lower-lying rotational and Zeeman levels (we assume that the collision energy is small enough so that rotational excitation of $\mathrm{CaH}$ into higher rotational manifolds $(N \geq 2)$ is energetically forbidden).

The state-to-state cross sections for rotational-Zeeman relaxation of ${ }^{40} \mathrm{CaH}$ molecules in a cold ${ }^{4} \mathrm{He}$ buffer gas follow a clear trend. The largest cross sections are those for spinconserving rotational relaxation $|8\rangle \rightarrow|2\rangle$ or $\left|11 \frac{1}{2}\right\rangle \rightarrow\left|00 \frac{1}{2}\right\rangle$. The second most efficient are the processes $|8\rangle \rightarrow|6\rangle\left(\left|11 \frac{1}{2}\right\rangle \rightarrow\left|1-1 \frac{1}{2}\right\rangle\right)$ and $|8\rangle \rightarrow|7\rangle\left(\left|11 \frac{1}{2}\right\rangle \rightarrow\left|10 \frac{1}{2}\right\rangle\right)$ that conserve $N$ and $M_{S}$ but change $M_{N}$. The slowest transitions are those which conserve $N$ but change $M_{S}:|8\rangle \rightarrow|5\rangle\left(\left|11 \frac{1}{2}\right\rangle \rightarrow\left|1-1-\frac{1}{2}\right\rangle\right),|8\rangle \rightarrow|4\rangle\left(\left|11 \frac{1}{2}\right\rangle \rightarrow\left|10-\frac{1}{2}\right\rangle\right)$, and, to a less extent, $|8\rangle \rightarrow|3\rangle\left(\left|11 \frac{1}{2}\right\rangle \rightarrow\left|11-\frac{1}{2}\right\rangle\right)$. 
Remarkably, as shown in Fig. 3, the slowest relaxation transition $|8\rangle \rightarrow|5\rangle$ occurs between the pure spin-stretched states $\left|11 \frac{1}{2}\right\rangle$ and $\left|1-1-\frac{1}{2}\right\rangle$. Note that all other $N=1$ states $(|3\rangle$, $|4\rangle,|6\rangle$, and $|7\rangle)$ are not pure $\left|N M_{N} M_{S}\right\rangle$ states, i.e., they have a non-negligible admixture of the $\left|N^{\prime} M_{N}^{\prime} M_{S}^{\prime}\right\rangle$ components with $M_{N}^{\prime} \neq M_{N}$ and $M_{S}^{\prime} \neq M_{S}$ due to the spin-rotation interaction (see above). As a result, transitions originating from state $|8\rangle$ to these "unpure" final states are not suppressed compared to the $|8\rangle \rightarrow|5\rangle$ transition.

\section{SUMMARY AND CONCLUSIONS}

We have demonstrated the possibility of computationally efficient quantum dynamical calculations of state-to-state scattering cross sections for cold molecular collisions in an external magnetic field. Prior to this work, such calculations have been hampered by the presence of unphysical states in the eigenspectrum of the asymptotic Hamiltonian in the total angular momentum representation [19-21, 29]. We have developed a general procedure for eliminating the unphysical states based on the observation that the asymptotic Hamiltonian commutes with the orbital angular momentum squared $\left(\hat{L}^{2}\right)$ of the collision complex. As a result, the asymptotic Hamiltonian is diagonal in the basis of simultaneous eigenstates of $\hat{L}^{2}$ and $\hat{J}^{2}$, i.e., the SF total angular momentum eigenstates. Using this property, we determined that the origin of unphysical states in molecular Zeeman spectra can be traced back to the incompleteness of fixed-l angular momentum basis sets, which do not have a sufficient number of $J$-states ranging from $|j-l|$ to $j+l$, leading in turn to an incomplete representation of the asymptotic Hamiltonian.

Armed with this insight, we developed a general procedure for removing the unphysical states from the spectrum of the asymptotic Hamiltonian. The procedure extends the number of $J$ states for each $l$ to include the complete set of quantum numbers ranging from $J=$ $|j-l|$ to $J=J+l$ as required by the angular momentum addition rules. This procedure can be implemented for each individual rotational state in the basis set, and ensures the completeness of the SF total angular momentum basis for each $l$, thereby keeping only the physical eigenstates in the spectrum. We have verified, through rigorous quantum scattering calculations of cold ${ }^{4} \mathrm{He}+{ }^{40} \mathrm{CaH}$ collisions, that the augmented SF total angular momentum basis provides accurate state-to-state scattering cross sections for all final states of ${ }^{40} \mathrm{CaH}$ over the range of collision energies $0.01-3 \mathrm{~K}$. 
The augmented SF total angular momentum basis proposed in this work removes the congestion caused by unphysical Zeeman states in the spectrum of the asymptotic Hamiltonian, thereby facilitating quantum scattering calculations of state-to-state cross sections and possibly near-threshold energy levels of strongly anisotropic atom-molecule collision complexes in the presence of an external magnetic field. Rigorous calculations of such spectra play a central role in the identification and assignment of magnetic Feshbach resonances in ultracold atom-molecule [23, 40, 41] and molecule-molecule [18, 42] collisions.

Because our procedure relies on the conservation of the orbital angular momentum of the collision complex in the limit $R \rightarrow \infty$, it is not directly applicable to the BF total angular momentum representation, where the operator $\hat{L}^{2}$ is not diagonal. In future work, it would be desirable to extend our procedure to eliminate the unphysical states directly in the $\mathrm{BF}$ representation. It would also be interesting to apply the procedure to more complex molecular states of, e.g., ${ }^{3} \Sigma$ and ${ }^{2} \Pi$ symmetries and to molecular collisions in the presence of an external electric field [38].

\section{ACKNOWLEDGEMENTS}

This work was supported by the NSF (Grant No. PHY-1912668).

\section{DATA AVAILABILITY STATEMENT}

The data that support the findings of this study are available from the corresponding author upon reasonable request.

[1] J. L. Bohn, A. M. Rey, and J. Ye, Cold molecules: Progress in quantum engineering of chemistry and quantum matter, Science 357, 1002 (2017).

[2] N. Balakrishnan and A. Dalgarno, Chemistry at ultracold temperatures, Chem. Phys. Lett. 341, 652 (2001).

[3] T. V. Tscherbul and R. V. Krems, Tuning bimolecular chemical reactions by electric fields, Phys. Rev. Lett. 115, 023201 (2015). 
[4] R. Hermsmeier, J. Kłos, S. Kotochigova, and T. V. Tscherbul, Quantum spin state selectivity and magnetic tuning of ultracold chemical reactions of triplet alkali-metal dimers with alkalimetal atoms, Phys. Rev. Lett. 127, 103402 (2021).

[5] J. F. E. Croft and J. L. Bohn, Long-lived complexes and chaos in ultracold molecular collisions, Phys. Rev. A 89, 012714 (2014).

[6] Y. Liu, M.-G. Hu, M. A. Nichols, D. D. Grimes, T. Karman, H. Guo, and K.K. Ni, Photo-excitation of long-lived transient intermediates in ultracold reactions, Nat. Phys. 16, 1132 (2020).

[7] A. Devolder, P. Brumer, and T. V. Tscherbul, Complete quantum coherent control of ultracold molecular collisions, Phys. Rev. Lett. 126, 153403 (2021).

[8] T. Bilitewski, L. De Marco, J.-R. Li, K. Matsuda, W. G. Tobias, G. Valtolina, J. Ye, and A. M. Rey, Dynamical generation of spin squeezing in ultracold dipolar molecules, Phys. Rev. Lett. 126, 113401 (2021).

[9] J. F. E. Croft, C. Makrides, M. Li, A. Petrov, B. K. Kendrick, N. Balakrishnan, and S. Kotochigova, Universality and chaoticity in ultracold $\mathrm{K}+\mathrm{KRb}$ chemical reactions, Nat. Commun. 8, 15897 (2017).

[10] D. DeMille, Quantum computation with trapped polar molecules, Phys. Lev. Lett. 88 (2002).

[11] A. André, D. DeMille, J. M. Doyle, M. D. Lukin, S. E. Maxwell, P. Rabl, R. J. Schoelkopf, and P. Zoller, A coherent all-electrical interface between polar molecules and mesoscopic superconducting resonators, Nat. Phys. 2, 636 (2006).

[12] S. F. Yelin, K. Kirby, and R. Côté, Schemes for robust quantum computation with polar molecules, Phys. Rev. A 74, 050301 (2006).

[13] A. V. Gorshkov, S. R. Manmana, G. Chen, E. Demler, M. D. Lukin, and A. M. Rey, Quantum magnetism with polar alkali-metal dimers, Phys. Rev. A 84, 033619 (2011).

[14] K. R. A. Hazzard, S. R. Manmana, M. Foss-Feig, and A. M. Rey, Far-from-equilibrium quantum magnetism with ultracold polar molecules, Phys. Rev. Lett. 110, 075301 (2013).

[15] A. Volpi and J. L. Bohn, Magnetic-field effects in ultracold molecular collisions, Phys. Rev. A 65, 052712 (2002).

[16] R. V. Krems and A. Dalgarno, Quantum-mechanical theory of atom-molecule and molecular collisions in a magnetic field: Spin depolarization, J. Chem. Phys. 120, 2296 (2004). 
[17] T. V. Tscherbul, G. C. Groenenboom, R. V. Krems, and A. Dalgarno, Dynamics of OH( $\left({ }^{2} \Pi\right)-\mathrm{He}$ collisions in combined electric and magnetic fields, Faraday Discuss. 142, 127 (2009).

[18] T. V. Tscherbul, Y. V. Suleimanov, V. Aquilanti, and R. V. Krems, Magnetic field modification of ultracold molecule-molecule collisions, New J. Phys. 11, 055021 (2009).

[19] T. V. Tscherbul and A. Dalgarno, Quantum theory of molecular collisions in a magnetic field: Efficient calculations based on the total angular momentum representation, J. Chem. Phys. 133, 184104 (2010).

[20] T. V. Tscherbul, J. Kłos, and A. A. Buchachenko, Ultracold spin-polarized mixtures of ${ }^{2} \Sigma$ molecules with $S$-state atoms: Collisional stability and implications for sympathetic cooling, Phys. Rev. A 84, 040701 (2011).

[21] Y. V. Suleimanov, T. V. Tscherbul, and R. V. Krems, Efficient method for quantum calculations of molecule-molecule scattering properties in a magnetic field, J. Chem. Phys. 137, 024103 (2012).

[22] W. C. Campbell, T. V. Tscherbul, H.-I. Lu, E. Tsikata, R. V. Krems, and J. M. Doyle, Mechanism of collisional spin relaxation in ${ }^{3} \Sigma$ molecules, Phys. Rev. Lett. 102, 013003 (2009).

[23] M. T. Hummon, T. V. Tscherbul, J. Kłos, H.-I. Lu, E. Tsikata, W. C. Campbell, A. Dalgarno, and J. M. Doyle, Cold $\mathrm{N}+\mathrm{NH}$ collisions in a magnetic trap, Phys. Rev. Lett. 106, 053201 (2011).

[24] A. Bergeat, J. Onvlee, C. Naulin, A. van der Avoird, and M. Costes, Quantum dynamical resonances in low-energy $\mathrm{CO}(j=0)+$ He inelastic collisions, Nat. Chem. 7, 349 (2015).

[25] S. N. Vogels, J. Onvlee, S. Chefdeville, A. van der Avoird, G. C. Groenenboom, and S. Y. T. van de Meerakker, Imaging resonances in low-energy NO-He inelastic collisions, Science 350, $787(2015)$.

[26] A. Bergeat, S. Chefdeville, M. Costes, S. B. Morales, C. Naulin, U. Even, J. Kłos, and F. Lique, Understanding the quantum nature of low-energy $\mathrm{C}\left({ }^{3} P_{j}\right)+$ He inelastic collisions, Nat. Chem. 10, 519 (2018).

[27] A. Klein, Y. Shagam, W. Skomorowski, P. S. Żuchowski, M. Pawlak, L. M. C. Janssen, N. Moiseyev, S. Y. T. van de Meerakker, A. van der Avoird, C. P. Koch, and E. Narevicius, Directly probing anisotropy in atom-molecule collisions through quantum scattering resonances, Nat. Phys. 13, 35 (2017). 
[28] S. N. Vogels, T. Karman, J. Kłos, M. Besemer, J. Onvlee, A. van der Avoird, G. C. Groenenboom, and S. Y. T. van de Meerakker, Scattering resonances in bimolecular collisions between NO radicals and $\mathrm{H}_{2}$ challenge the theoretical gold standard, Nat. Chem. 10, 435 (2018).

[29] M. Morita, M. B. Kosicki, P. S. Żuchowski, and T. V. Tscherbul, Atom-molecule collisions, spin relaxation, and sympathetic cooling in an ultracold spin-polarized $\operatorname{Rb}\left({ }^{2} S\right)-\operatorname{SrF}\left({ }^{2} \Sigma^{+}\right)$ mixture, Phys. Rev. A 98, 042702 (2018).

[30] M. Morita, J. Kłos, and T. V. Tscherbul, Full-dimensional quantum scattering calculations on ultracold atom-molecule collisions in magnetic fields: The role of molecular vibrations, Phys. Rev. Research 2, 043294 (2020).

[31] M. Lara, J. L. Bohn, D. Potter, P. Soldán, and J. M. Hutson, Ultracold Rb-OH collisions and prospects for sympathetic cooling, Phys. Rev. Lett. 97, 183201 (2006).

[32] A. O. G. Wallis and J. M. Hutson, Production of ultracold NH molecules by sympathetic cooling with Mg, Phys. Rev. Lett. 103, 183201 (2009).

[33] Z. Pavlovic, T. V. Tscherbul, H. R. Sadeghpour, G. C. Groenenboom, and A. Dalgarno, Cold collisions of $\mathrm{OH}\left({ }^{2} \Pi\right)$ molecules with $\mathrm{He}$ atoms in external fields, J. Phys. Chem. A 113, 14670 (2009).

[34] P. S. Żuchowski and J. M. Hutson, Cold collisions of $\mathrm{N}\left({ }^{4} \mathrm{~S}\right)$ atoms and $\mathrm{NH}\left({ }^{3} \Sigma\right)$ molecules in magnetic fields, Phys. Chem. Chem. Phys. 13, 3669 (2011).

[35] T. V. Tscherbul and R. V. Krems, Controlling electronic spin relaxation of cold molecules with electric fields, Phys. Rev. Lett. 97, 083201 (2006).

[36] M. Morita, J. Kłos, A. A. Buchachenko, and T. V. Tscherbul, Cold collisions of heavy ${ }^{2} \Sigma$ molecules with alkali-metal atoms in a magnetic field: Ab initio analysis and prospects for sympathetic cooling of $\operatorname{SrOH}\left({ }^{2} \Sigma^{+}\right)$by $\operatorname{Li}\left({ }^{2} S\right)$, Phys. Rev. A 95, 063421 (2017).

[37] O. Dulieu and A. Osterwalder, eds., Cold Chemistry: Molecular Scattering and Reactivity Near Absolute Zero (Royal Society of Chemistry, 2018).

[38] T. V. Tscherbul, Total-angular-momentum representation for atom-molecule collisions in electric fields, Phys. Rev. A 85, 052710 (2012).

[39] A. M. Arthurs and A. Dalgarno, The theory of scattering by a rigid rotator, Proc. R. Soc. London Ser. A 256, 540 (1960).

[40] T. V. Tscherbul, J. Kłos, L. Rajchel, and R. V. Krems, Fine and hyperfine interactions in cold YbF-He collisions in electromagnetic fields, Phys. Rev. A 75, 033416 (2007). 
[41] X.-Y. Wang, M. D. Frye, Z. Su, J. Can, L. Liu, D.-C. Zhang, G. Yang, J. M. Hutson, B. Zhao, C.-L. Bai, and J.-W. Pan, Magnetic Feshbach resonances in collisions of ${ }^{23} \mathrm{Na}^{40} \mathrm{~K}$ with ${ }^{40} \mathrm{~K}$, arXiv:2103.07130v1 (2021).

[42] Y. V. Suleimanov and R. V. Krems, Efficient numerical method for locating feshbach resonances of ultracold molecules in external fields, J. Chem. Phys. 134, 014101 (2011).

[43] R. N. Zare, Angular Momentum (Wiley, 1988).

[44] G. C. Groenenboom and N. Balakrishnan, The He-CaH $\left({ }^{2} \Sigma^{+}\right)$interaction. I. Three-dimensional ab initio potential energy surface, J. Chem. Phys. 118, 7380 (2003).

[45] R. V. Krems, A. Dalgarno, N. Balakrishnan, and G. C. Groenenboom, Spinflipping transitions in ${ }^{2} \Sigma$ molecules induced by collisions with structureless atoms, Phys. Rev. A 67, 060703 (2003).

[46] N. Balakrishnan, G. C. Groenenboom, R. V. Krems, and A. Dalgarno, The He-CaH $\left({ }^{2} \Sigma^{+}\right)$interaction. II. Collisions at cold and ultracold temperatures, J. Chem. Phys. 118, 7386 (2003).

[47] T. V. Tscherbul and R. V. Krems, Manipulating spin-dependent interactions in rotationally excited cold molecules with electric fields, J. Chem. Phys. 125, 194311 (2006).

[48] E. Abrahamsson, T. V. Tscherbul, and R. V. Krems, Inelastic collisions of cold polar molecules in nonparallel electric and magnetic fields, J. Chem. Phys. 127, 044302 (2007). 